\title{
The Impact of Occlusion on in vivo Skin Integrity Recovery
}

\author{
Acerca do Impacto da Oclusão na Reparação in vivo da Integridade Cutânea
}

\author{
Maria Madalena Pereira ${ }^{1}$, Joana Saraiva ${ }^{1}$, Luís Monteiro Rodrigues ${ }^{1,2}$ \\ ${ }^{1}$ CBIOS-UDE, Research Center for Health Science and Technologies, Universidade Lusófona. \\ ${ }^{2}$ Experimental Physiology Lab, Universidade de Lisboa, (Fac Farmácia) Portugal \\ E-mail:madalena.pereira@gmail.com
}

\begin{abstract}
The wide diversity of wound dressings available to recover skin integrity makes the selection a complex and difficult process. It is crucial to learn more about the specific impact of different wound dressings. The objective of the present study is to illustrate the impact of the occlusion on the recovery of the skin "barrier" function. Eight healthy women $(\overline{\mathbf{x}}=22,6 \pm 1,1)$ were chosen and three experimental sites marked on the ventral aspect of both forearms, in order to apply a skin surface biopsy (SSB) with cyanoacrylate. An additional site was not submitted to SSB and thus used as a negative control (NC). Two of the SSB sites were randomly occluded with hydroxypolyurethane (PermaFoam ${ }^{\circledR}$, Hartmann) (site A) or with parafilm (site B) and the third site (site C) was left without occlusion, acting as a positive control (PC). The variables studied were the Trans-epidermal water loss (TEWL, Tewameter TM300), the erythema (Minolta CR3000) and local microcirculation (LDF Periflux).

Results suggest a faster recovery of skin integrity in all the occluded sites, particularly when using the hydroxypolyurethane wound dressing, compared with the control group. We conclude that, under these experimental conditions, occlusion determines a greater and faster recovery of skin integrity in vivo.
\end{abstract}

Keywords: epidermal skin barrier; repair; skin surface biopsy; occlusion, hydroxypolyurethane.

\begin{abstract}
Resumo
A grande diversidade de apósitos disponível para a recuperação da integridade cutânea torna complexa e difícil a sua escolha. Torna-se pois fundamental, aprofundar o conhecimento sobre o impacto específico dos diferentes materiais de penso. O presente estudo pretende ilustrar o impacto da oclusão na recuperação da função de "barreira" da pele e envolveu 8 mulheres saudáveis $(\overline{\mathbf{x}}=22,6 \pm 1,1)$ em cujos antebraços foram marcados 3 sítios experimentais sujeitos a biopsia superficial cutânea (BSC) com cianoacrilato, mantendo integro um quarto local, assim utilizado como controlo negativo $(\mathrm{CN})$. Dois dos locais experimentais sujeitos a BSC foram aleatoriamente ocludidos com o apósito de hidroxipoliuretano (PermaFoam ${ }^{\circledR}$, Hartmann)(sitio A) ou com parafilm (sitio B) e o terceiro local (sitio C) deixado sem oclusão, funcionando como controlo positivo (CP). As variáveis em estudo foram a Perda Transepidérmica de água (PTEA, Tewameter TM300), o eritrema (Minolta CR3000) e a microcirculação local (LDF Periflux).

Os resultados sugerem uma mais rápida recuperação da integridade cutânea nos sítios ocludidos, quando comparados com o respectivo controle, salientando-se nestes, o local tratado com o apósito de hidroxipoliuretano. Conclui-se que, nas presentes condições experimentais, a oclusão determina uma maior e mais rápida recuperação da integridade cutânea in vivo.
\end{abstract}

Palavras-chave: função "barreira" epidérmica; reparação; Biopsia Superficial Cutânea; oclusão; hidroxipoliuretano 


\section{Introduction}

The skin lesion recovery process is highly complex requiring a multidisciplinary approach and continuous research in order to improve therapeutical interventions on patient care.

The central purpose of the cutaneous healing is to recover the so called "barrier" function, while ensuring the organ's continuity which involves a large number and variety of cellular and molecular players ${ }^{[1-5]}$. It is a complex process, and many solutions have been designed to improve it, including the development of better and more effective wound dressings. However, occlusion itself is regarded as essential to provide an environment favoring the tissue repair ${ }^{[6-13]}$. The continual development of new dressing materials makes their classification and appropriate selection difficult .Moreover, only a few studies address those aspects related to the efficacy of these devices. ${ }^{[14,15]}$. In the present study we tried to develop a model to highlight the role of occlusion in the recovery of the skin's integrity in vivo. The study focused on the epidermal "barrier" function, and at the same time, assessed the impact of the hydroxypolyurethane dressing which is one of the most used in clinical practice, after skin surface biopsy (SSB).

\section{Material and Methods}

A convenience sample was chosen consisting of eight individuals, all female, aged between 20 and $25(\overline{\mathbf{x}}=$ $22,6 \pm 1,1)$.Volunteers were selected after informed consent, according to previously established inclusion criteria and fully compliant with all ethical standards set by the Declaration of Helsinki and respective amendments ${ }^{[16-19]}$.

To address the research questions, a method using a SSB procedure, with cyanoacrylate (Super Glue, China) ${ }^{[20-23]}$ was applied. This method allowed us to modify, in a controlled way, the epidermal "barrier" function, and monitor the evolution of the recovery process.

The ventral aspect of both forearms was used. For each volunteer ,four experimental sites were selected, two on each forearm, which were symmetrical and randomly chosen. After randomization (Latin square) three experimental sites were subjected to SSB, and a fourth place was left intact, being the negative control (NC). Two of the experimental sites submitted to SSB were randomly occluded with the hydroyipolyurethane (PermaFoam ${ }^{\circledR}$, Hartmann) (site A) or with parafilm (site B) and the third site (site C) was left without occlusion, acting as the positive control (PC).

$\mathrm{Th}$ e chose $\mathrm{n}$ variables were : - Trans-epidermal water loss (TEWL), obtained by evaporimetry (TM300 Tewameter CK electronics,

\section{Introdução}

O processo de recuperação da lesão cutânea reveste-se de elevada complexidade, havendo necessidade de uma intervenção multidisciplinar bem como de uma investigação continuada, visando a melhoria da eficácia de intervenção no paciente .

O mecanismo que envolve a reparação da lesão cutânea tem como objectivo central a recuperação das propriedades de "barreira" da pele, para além de assegurar a continuidade do mesmo, e envolve elevado número e variedade de intervenientes celulares e moleculares ${ }^{[1-5]}$. Trata-se de um processo complexo, para o qual procuram contribuir outras soluções, designadamente através do desenvolvimento de material de penso cada vez mais eficaz, uma vez que se assume que a oclusão só por si, é susceptível de proporcionar um ambiente especialmente propício à reparação tecidular ${ }^{[6-13]}$.

O constante desenvolvimento de novos materiais de penso tem dificultado a sua classificação e, consequentemente, o processo de escolha. Esta tarefa é ainda dificultada pelo reduzido numero de estudos que abordam a eficácia destes dispositivos ${ }^{[14,15]}$. Assim, no presente trabalho, procurámos desenvolver um modelo de estudo que nos permitisse evidenciar o papel da oclusão sobre a recuperação da integridade cutânea in vivo, focando a função de "barreira" epidérmica e, ao mesmo tempo, avaliar o impacto de um apósito de hidroxipoliuretano, um dos mais utilizados na prática clínica, após biopsia superficial cutânea (BSC).

\section{Material e Métodos}

Foi utilizada uma amostra de conveniência constituída por 8 indivíduos, do sexo feminino, com idades compreendidas entre 20 e os 25 anos ( $\quad \overline{\mathbf{x}} 22,6 \pm 1,1)$. Os voluntários foram seleccionados após consentimento informado, de acordo com critérios de inclusão previamente estabelecidos e no pleno respeito de todas as normas éticas previstas pela Declaração de Helsínquia e respectivas emendas ${ }^{[16-19]}$.

Para abordar a questão de investigação, foi utilizado um micrométodo de estudo in vivo através de BSC com cianoacrilato (Super Glue, China) ${ }^{[20-23]}$. Este método permitiu alterar, de forma controlada, a função de barreira da pele, possibilitando quer a avaliação das alterações ocorridas quer a sua monitorização no processo de recuperação.

A área anatómica escolhida foi a região central da face anterior do antebraço por ser um local quase desprovido de pilosidade e ser de fácil acesso. Em cada voluntário foram seleccionados quatro sítios experimentais, dois em cada antebraço de forma simétrica. Após aleatorização (quadrado latino) 3 sítios experimentais foram sujeitos a BSC com cianoacrilato, e um quarto local não sujeito a $\mathrm{BSC}$ funcionou como controlo negativo $(\mathrm{CN})$. Dois dos 
Germany) and expressed in g/h.m2. The TEWL is regarded as the best quantitative indicator to assess epidermal "barrier" properties ${ }^{[2426]}$;

- the a* parameter detected by colorimetry (Chromameter ${ }^{\circledR}$ CR 300, Minolta, Japan), expressed in arbitrary units (AU). Several studies show the relationship of this variable with the cutaneous erythema, and to the inflammatory response of skin to various stimuli ${ }^{[25]}$;

- local microcirculation measured by laser Doppler flowmetry (Periflux ${ }^{\circledR}$ PF5010, Perimed, Sweden), expressed in arbitrary perfusion units (BPU's). The change in skin integrity may cause changes in local microcirculation ${ }^{[26]}$.

All variables were determined at baseline, 5 minutes (D0), 3 days (D3), 6 days (D6), 10 days (D10) and 14 days (D14) after SSB.

The dressing under study was applied until the values $\square \square$ of TEWL, used as the statistical "end point", reached full stabilization. This variable is considered the indicator of the skin "barrier" function ${ }^{[24]}$.

Evaluations were made in the laboratory under controlled temperature and humidity $\left(21 \pm 1^{\circ} \mathrm{C}, 45 \pm\right.$ $5 \%$ ), in the absence of heat sources and forced convection, according to previously published methodology ${ }^{[25]}$.

Data analysis included descriptive and comparative statistics using the SPSS (version 14.0 for Windows) and Microsoft Excel \& 2003. The comparative analysis was performed using the nonparametric Friedman and Wilcoxon test for paired data, adopting a significance level of $95 \%$.

\section{Results and Discussion}

The individual biometric characteristics of the studied subjects are presented in the next table (Table I).

Regarding mean values, we observed that the maximum value was reached within 5 minutes after the SSB procedure for all variables. It is especially significant that the TEWL increase, as noted before, is a good indicator of skin's integrity. The local microcirculation and chromaticity related to the erythema $\left(\mathrm{a}^{*}\right)$ modifications are suggestive of local inflammatory response related to the applied procedure ${ }^{[26-29]}$.

In order to compare the subject's skin functional performance we first tested baseline values from all experimental sites for each of the biometric techniques used. The obtained $\mathrm{p}$ values (TEWL: 0.440 , a $*: 0.392$ and cutaneous microcirculation: 0.782 ) show that the functional status of the skin was similar in all individuals.

We also compared the experimental site D performance, used as negative control, for all measurement periods in order to evaluate the eventual locais experimentais sujeitos a BSC foram aleatoriamente ocludidos com o apósito de hidroxipoliuretano (PermaFoam ${ }^{\circledR}$, Hartmann)(sitio A) ou com parafilm (sitio B) e o terceiro local (sitio C) foi deixado sem oclusão, funcionando como controlo positivo (CP).

As variáveis quantificadas foram:

- a perda trans-epidérmica de água, obtida por evaporimetria (Tewameter TM300 CK electronics, Germany), expressa em g/h.m2. A PTEA é considerada como o melhor indicador quantitativo na avaliação das propriedades de "barreira" cutânea ${ }^{[24-26]}$;

- o parâmetro a* obtido por colorimetria (Chromameter ${ }^{\circledR}$ CR 300, Minolta, Japan), expresso em unidades arbitrárias (U.A.). Vários estudos demonstram a relação desta variável com o eritema cutâneo relacionando-o com a resposta inflamatória da pele a diversos estímulos ${ }^{[25]}$;

- a microcirculação local medida por fluxometria de laserdoppler, expressa em unidades arbitrárias de perfusão (BPU's) (Periflux ${ }^{\circledR}$ PF5010, Perimed, Suécia). A alteração da integridade cutânea pode provocar alterações na microcirculação local ${ }^{[2]}$.

Todas as variáveis foram determinadas em condições basais, 5 minutos (D0), 3 dias (D3), 6 dias (D6), 10 dias (D10) e 14 dias (D14) após BSC.

O material de penso em estudo foi mantido até regularização dos valores de PTEA, assim utilizado como "end point" estatístico, uma vez que esta variável é considerada como o indicador de função de "barreira" cutânea ${ }^{[24]}$.

As avaliações foram efectuadas em ambiente laboratorial, em condições de temperatura e humidade controladas $(21 \pm$ $1^{\circ} \mathrm{C} ; 45 \pm 5 \%$ ), na ausência de fontes de calor e de convecção forçada, de acordo com metodologia e recomendações previamente publicadas ${ }^{[25]}$.

$\mathrm{O}$ tratamento dos dados incluiu estatística descritiva e estatística comparativa recorrendo aos programas SPSS (versão 14.0 para Windows) e Microsoft Excel ${ }^{\circledR} 2003$. A análise comparativa foi realizada através dos testes não paramétricos de Friedman e de Wilcoxon para dados emparelhados, adoptando-se um nível de significância de $95 \%$.

\section{Resultados e Discussão}

Os resultados relativos à caracterização biométrica dos voluntários estão sistematizados na tabela seguinte (Tabela I).

Relativamente à média dos valores analisados podemos observar que o valor máximo foi atingido 5 minutos após a BSC para todas as variáveis. É especialmente revelador o aumento da PTEA considerado como um bom indicador da integridade cutânea. A alteração dos valores de microcirculação local e da cromaticidade relacionada com o eritema $\left(\mathrm{a}^{*}\right),{ }^{[26-29]}$ são indiciadores de resposta inflamatória local, em função da técnica aplicada.

Para comparar o comportamento funcional cutâneo dos indivíduos estudados comparámos, em primeiro lugar, os valores basais obtidos em todos os sítios experimentais e para cada uma das técnicas biométricas utilizadas. Os valores de p_value(PTEA:0,440, a*:0,392 e 
interference of external factors (environmental and instrumental) on results. The $\mathrm{p}$ value obtained (TEWL: 0.493 , a *: 0.287 and cutaneous microcirculation: 0.754 ) confirmed that the measurement conditions were similar on the different days, and did not influence results.

The relative impact of the procedure was also analyzed in all experimental sites prolonging the analysis for another 5 minutes after the SSB. Obtained p_values (TEWL: 0.223, a *: cutaneous microcirculation and 0.419: 0.882) suggest that all experimental sites responded similarly to the SSB. microcirculação cutânea: 0,782 ) revelam que o comportamento funcional cutâneo na amostra estudada era semelhante em todos os voluntários.

Comparámos ainda o comportamento funcional do sítio experimental D, utilizado como controlo negativo, em todos os momentos de medição, para averiguar a eventual interferência de factores externos (ambientais e relacionados com os instrumentos) nos resultados obtidos. Os valores de $p$ value encontrados (PTEA:0,493, a*:0,287 e microcirculação cutânea: $0,754)$ revelam que as condições de determinação dos diferentes valores foram semelhantes nos diferentes dias do estudo, não influenciando os resultados.

Procurámos ainda comparar o impacto relativo do procedimento nos diferentes sítios experimentais, prolongando ainda a análise $5 \mathrm{~min}$ após a BSC. Os valores de $p$ value encontrados (PTEA:0,223, a*:0,419 e microcirculação cutânea: 0,882 ) sugerem que todos os locais experimentais responderam de forma semelhante à BSC.

Table 1 - mean and standard deviation of TEWL ( $\mathrm{gh} / \mathrm{m} 2)$, cutaneous microcirculation (BPU) and skin Colorimetry (a *) (AU) in basal conditions, 5 minutes (D0), 3 (D3), 6 (D6) 10 (D10) e 14 (D14) days after SSC) in eight volunteers. (A - Hydroxypolyurethane, B - Parafilm, C positive control: $\mathrm{D}$ - negative control.

Tabela 1 - Média e desvio padrão de PTEA (g.h/m2), Microcirculação cutânea (BPU) e Colorimetria cutânea (a*) (UA) em condições basais e após 5 minutos (D0), 3 (D3), 6 (D6), 10 (D10) e 14 (D14) dias após BSC nos oito voluntários. (A - Apósito de poliuretano; B Apósito de Parafilm; C-Controlo positivo; D-Controlo negativo).

\begin{tabular}{|c|c|c|c|c|c|c|c|}
\hline \multirow{2}{*}{$\begin{array}{l}\text { Variables } \\
\text { Variável }\end{array}$} & \multirow{2}{*}{$\begin{array}{l}\text { Sites } \\
\text { Sítios }\end{array}$} & \multicolumn{6}{|c|}{$\begin{array}{l}\text { Mean } \pm \text { standard deviation in the various stages of evaluation } \\
\text { Média } \pm \text { desvio padrão nos vários momentos de avaliação }\end{array}$} \\
\hline & & Basal & D0 & D3 & D6 & D10 & D14 \\
\hline \multirow{4}{*}{$\begin{array}{c}\text { TEWL } \\
\text { PTEA }\left(g / h \cdot m^{2}\right)\end{array}$} & A & $9,0 \pm 3,4$ & $44,6 \pm 21,5$ & $24,4 \pm 14,9$ & $14,7 \pm 8,3$ & $11,3 \pm 4,1$ & $9,8 \pm 3,4$ \\
\hline & B & $8,7 \pm 3,2$ & $28,5 \pm 14,0$ & $18,4 \pm 5,7$ & $16,2 \pm 5,6$ & $17,6 \pm 8,3$ & $12,8 \pm 7,1$ \\
\hline & C & $9,1 \pm 3,7$ & $39,0 \pm 18,3$ & $22,5 \pm 10,4$ & $13,7 \pm 5,0$ & $14,0 \pm 7,9$ & $11,2 \pm 4,3$ \\
\hline & D & $8,0 \pm 3,3$ & $8,0 \pm 3,3$ & $9,2 \pm 3,3$ & $8,5 \pm 1,9$ & $9,1 \pm 2,2$ & $8,6 \pm 2,4$ \\
\hline \multirow{4}{*}{$\begin{array}{l}\text { Local Flow } \\
\text { Fluxo Local } \\
\text { (BPU) }\end{array}$} & $\mathbf{A}$ & $6,7 \pm 2,3$ & $50,8 \pm 41,6$ & $57,2 \pm 88,3$ & $25,4 \pm 32,4$ & $15,9 \pm 12,6$ & $11,6 \pm 4,6$ \\
\hline & B & $8,8 \pm 1,8$ & $34,1 \pm 22,2$ & $32,5 \pm 18,8$ & $20,6 \pm 9,4$ & $17,2 \pm 6,3$ & $13,2 \pm 3,8$ \\
\hline & C & $6,9 \pm 1,1$ & $59,5 \pm 53,0$ & $28,6 \pm 21,7$ & $14,2 \pm 7,4$ & $10,2 \pm 3,3$ & $8,6 \pm 1,8$ \\
\hline & D & $8,1 \pm 2,4$ & $8,1 \pm 2,4$ & $7,1 \pm 1,5$ & $7,4 \pm 1,4$ & $7,3 \pm 2,0$ & $8,8 \pm 2,6$ \\
\hline \multirow{4}{*}{$\begin{array}{l}\text { Chromaticity } \\
\text { Colorimetria } \\
\left(a^{*}\right)(\text { UA) }\end{array}$} & A & $7,0 \pm 1,3$ & $11,8 \pm 4,4$ & $11,0 \pm 5,3$ & $9,7 \pm 4,6$ & $9,1 \pm 3,3$ & $8,1 \pm 1,8$ \\
\hline & B & $6,8 \pm 1,5$ & $12,3 \pm 4,8$ & $10,1 \pm 2,4$ & $11,2 \pm 2,7$ & $10,2 \pm 2,7$ & $8,9 \pm 2,2$ \\
\hline & C & $7,0 \pm 1,8$ & $11,8 \pm 2,2$ & $10,0 \pm 2,3$ & $9,6 \pm 2,1$ & $7,9 \pm 1,7$ & $8,1 \pm 1,1$ \\
\hline & D & $7,1 \pm 1,7$ & $7,1 \pm 1,7$ & $7,5 \pm 0,7$ & $7,1 \pm 0,9$ & $7,1 \pm 1,2$ & $7,1 \pm 1,6$ \\
\hline
\end{tabular}


Finally the skin "barrier" recovery process was compared in all experimental sites. Results are shown in Table II. For this objective, TEWL was used as the statistical "end point" since this variable is regarded as a reliable indicator of the epidermal "barrier" ${ }^{[24]}$. Several studies have demonstrated its usefulness, in both animal and human cases, following changes in barrier function after trauma from different origins ${ }^{[30]}$, and it can be used to monitor the therapeutical efficacy of different dressing materials ${ }^{[5,31,32]}$. TEWL results until D10 show that, statistically significant differences were detected in all experimental sites compared with the site $\mathrm{D}(\mathrm{NC})$. The experimental site treated with the hydroxypolyurethane dressing showed similar values to the baseline on day 10 (D10), meaning a faster recovery of the barrier function.
Procedemos, finalmente, ao estudo comparativo da capacidade de recuperação dos diferentes sítios experimentais. Os resultados podem ser observados na tabela II. Para tal, utilizámos a PTEA "end point" estatístico uma vez que esta variável é considerada como um indicador fiável da função "barreira" epidérmica ${ }^{[24]}$. Diversos estudos têm demonstrado a aplicabilidade desta variável, quer em modelos humanos quer em modelos animais, no estudo da integridade da pele após trauma de diferentes origens ${ }^{[30]}$, quer na monitorização da eficácia terapêutica de diferentes materiais de penso ${ }^{[5,31,32]}$. Os resultados obtidos para esta variável mostram que até D10 todos os sítios experimentais apresentavam diferenças estatisticamente significativas comparativamente ao sítio experimental D (CN). Por seu lado, o sítio, ocludido com o apósito de hidroxipoliuretano apresentou valores semelhantes aos valores basais a partir do dia 10 (D10) sugerindo uma mais rápida recuperação da função barreira.

Table 2 - Comparison of values obtained during several days of study for each technique biometric evaluations (pvalue obtained by Wilcoxon test $)(n=8)$

Tabela 2 - Comparação dos valores obtidos durante os vários dias do estudo para cada técnica de avaliação biométrica ( $\mathrm{p}$-value obtido através do teste de Wilcoxon) $(\mathrm{n}=8)$.

\begin{tabular}{|c|c|c|c|c|c|c|c|}
\hline \multirow[b]{2}{*}{$\begin{array}{l}\text { Variables } \\
\text { Variáveis }\end{array}$} & \multicolumn{7}{|c|}{ p value } \\
\hline & $\begin{array}{l}\text { Day } \\
\text { Dia }\end{array}$ & $\begin{array}{c}\text { Local A vs } \\
\text { Local B }\end{array}$ & $\begin{array}{c}\text { Local A vs } \\
\text { Local C }\end{array}$ & $\begin{array}{c}\text { Local A vs } \\
\text { Local D }\end{array}$ & $\begin{array}{c}\text { Local B vs } \\
\text { Local C }\end{array}$ & $\begin{array}{c}\text { Local B vs } \\
\text { Local D }\end{array}$ & $\begin{array}{c}\text { Local C vs } \\
\text { Local D }\end{array}$ \\
\hline \multirow{5}{*}{$\begin{array}{c}\text { TEWL } \\
\text { PTEA } \\
\text { (g/h.m2) }\end{array}$} & $\overline{\mathrm{D} 0}$ & 0,069 & 0,674 & 0,012 & 0,050 & 0,012 & 0,012 \\
\hline & D3 & 0,263 & 0,889 & 0,012 & 0,484 & 0,017 & 0,036 \\
\hline & D6 & 0,833 & 0,674 & 0,017 & 0,484 & 0,012 & 0,050 \\
\hline & D10 & 0,327 & 0,674 & 0,237 & 0,263 & 0,017 & 0,012 \\
\hline & D14 & 0,575 & 0,208 & 0,327 & 0,327 & 0,161 & 0,069 \\
\hline \multirow{5}{*}{$\begin{array}{c}\text { Local Flow } \\
\text { Fluxo Local } \\
\text { (B.P.U.) }\end{array}$} & D0 & 0,208 & 1,000 & 0,012 & 0,161 & 0,012 & 0,012 \\
\hline & D3 & 0,674 & 0,575 & 0,012 & 0,889 & 0,017 & 0,017 \\
\hline & $\overline{\mathrm{D} 6}$ & 0,779 & 0,575 & 0,017 & 0,123 & 0,017 & 0,017 \\
\hline & $\overline{\mathrm{D} 10}$ & 0,674 & 0,327 & 0,128 & 0,017 & 0,012 & 0,161 \\
\hline & D14 & 0,123 & 0,063 & 0,262 & 0,035 & 0,068 & 0,726 \\
\hline \multirow{5}{*}{$\begin{array}{l}\text { Chromaticity } \\
\text { Colorimetria } \\
\qquad \begin{array}{c}\left(\mathbf{a}^{*}\right) \\
\text { (U.A.) }\end{array}\end{array}$} & D0 & 0,401 & 0,575 & 0,012 & 0,674 & 0,017 & 0,012 \\
\hline & D3 & 0,327 & 0,779 & 0,017 & 0,123 & 0,012 & 0,025 \\
\hline & D6 & 0,674 & 0,575 & 0,012 & 0,889 & 0,017 & 0,017 \\
\hline & D10 & 0,401 & 0,401 & 0,123 & 0,036 & 0,017 & 0,208 \\
\hline & D14 & 0,779 & 0,674 & 0,093 & 0,484 & 0,208 & 0,069 \\
\hline
\end{tabular}


Some studies stressed the importance of local microcirculation evaluation in the inflammatory response after injury, ${ }^{[31]}$ since LDF enables the quantitative description of some important indicators such as the time of the recovery ${ }^{[32]}$. Our results reveal that occluded sites ( $A$ and $C$ ) reached the baseline on day 10 (D10), while site B (Parafilm) only recovered on day 14 (D14). Regarding the red chromaticity (a*) sites A (hydroxypolyurethane) and $\mathrm{C}$ (positive control) reached the baseline reference values on day 10 (D10) whereas the site B (parafilm) only fully recovered on day 14(D14).

Results clearly suggest that experimental sites submitted to SSB and covered with wound dressings (hydroxypolyurethane) and parafilm, recovered faster, demonstrating the importance of occlusion in the recovery of skin's integrity.

\section{Conclusions}

The micromodel developed here has allowed an objective approach to understanding the human in vivo cutaneous recovery process, under standard and controlled conditions. The biometric variables evaluated were adequate to describe the pathophysiological mechanisms involved, both in changing the epidermal "barrier" integrity, following the SSB procedure, and also during the recovery phase. Results suggest that, under the present experimental conditions, the skin's integrity recovered faster in the occluded sites, and in particular, in the site treated with the hydroxypoliurethane dressing, confirming the importance of occlusion in repairing the epidermal barrier function.

\section{Conflict of interests}

The authors declare that there are no financial and personal relationships that could be viewed as presenting a potential conflict of interests.
Alguns estudos têm salientado a importância da microcirculação local na avaliação da resposta inflamatória após lesão ${ }^{[31]}$ uma vez que a medida da LDF permite a quantificação de alguns indicadores funcionais como o tempo de recuperação da área ${ }^{[32]}$. Os nossos resultados revelam que os sítios ocludidos (A e C) atingiram os valores basais no dia 10 (D10), enquanto que o sitio B (Parafilme) apenas recuperou no dia 14(D14).

No que diz respeito à cromaticidade vermelha $\left(\mathrm{a}^{*}\right)$ os sitios A (apósito de hidroxipoliuretano) e C (controlo positivo) atingiram os valores basais no dia 10 (D0) enquanto que o sitio B (parafilm) apenas recuperou integralmente no dia 14.

Os resultados parecem sugerir que os sítios experimentais sujeitos a BSC e cobertos com o apósito em estudo (hidroxipoliuretano) e com parafilme, recuperaram mais rapidamente demonstrando a importância da oclusão na recuperação da integridade cutânea.

\section{Conclusões}

O micromodelo de estudo que aqui aplicámos permitiu uma abordagem objectiva do processo de recuperação da pele humana in vivo, em condições padronizadas e controladas. As variáveis biométricas avaliadas parecem ser adequadas à descrição dos mecanismos fisiopatológicos envolvidos quer na alteração da integridade cutânea, subjacente à $\mathrm{BSC}$, quer durante a fase de recuperação, permitindo um seguimento objectivo em ambas as fases do estudo experimental.

Os resultados revelam que, nas actuais condições experimentais, a recuperação da integridade cutânea foi mais rapidamente conseguida nos sítios cobertos e, em especial no sítio tratados com o apósito de hidroxipoliuretano, confirmando assim a importância da oclusão na reparação da função de barreira epidérmica.

\section{Conflito de Interesses}

Os autores declaram não existir qualquer relação pessoal ou financeira que possa ser entendida como representando um potencial conflito de interesses. 


\section{References / Referências}

[1] Kasting GB, Barai ND. Equilibrium water sorption in human stratum corneum. Journal of pharmaceutical sciences 2003; $92(8): 1624-1631$.

[2] Marks R. The stratum corneum barrier: the final frontier. J Nutr. 2004; 134(8):2017-2021.

[3] Baum CL, Arpey CJ. Normal cutaneous wound healing: clinical correlation with cellular and molecular events. American Society for Dermatologic Surgery 2005; 31: 674-686.

[4] Rodrigues LM, Roberto MA. Characterization strategies for the functional assessment of the cutaneous lesion. Burns. 2006; 32 (7): 797-801

[5] Pereira, MM. Estudo in vivo do impacto biológico de diferentes materiais de penso sobre a integridade cutânea. Diss Mest, FFUL, Lisboa 2008, 10-23.

[6] Harding KG, Moris HL, Patel GK. Healing chronic wounds. BMJ 2002; 324: 160-163

7] Barbara $\mathrm{P}$ et al. Wound prevalence, types, and treatments in Home care. Adv Wound Care 1999; 12 . 117-26.

[8] Trueman P, Posnett J. What price wound care?. The International Journal of Lower Extremity Wounds 2006; $5: 230-232$.

[9] Zhai H, Maibach HI. Occlusion vs Skin barrier function. Skin Research and technology 2002; 8: 1-6.

[10] Bradley $\mathrm{M}$ et al. Systematic reviews of wound care management: (2). Dressings and topical agents used in the healing of chronic wounds. Health Technol Assess 1999; 3 (17 Pt 2):1-35.

11] Zhai X, Yokota M, Maibach HI. In vitro human skin model to evaluate water permeability and determine wound dressings occlusivity. Cutan Ocul Toxicol 2007; 26(2): 107-111.

[12] João M, Proença e Cunha E. Material para o tratamento de feridas. In: Rocha $\mathrm{MJ}$ et al, eds. Feridas uma arte secular. 2nd. Coimbra: MinevaCoimbra 2006;
6: 161-209.

[13] Sholar AD et al. The specialized wound care center. A 7-year experience at a tertiary care hospital. Annals of plastic surgery $2007 ; 58(3): 279-284$.

[14] López JR et al. Valorción y atención integral a paciente com heridas. In: Agreda JJ, Bou JE eds. Atención integral de las heridas crónicas. Madrid: SPA, S.L. $2004 ; 1$ (4): 47-61

[15] Ovington LG. Advances in wound dressings. Clin Dermatol 2007; 25:33-38

[16] Diário da República, Lei 46/2004 de 19 de Agosto. [17]EUROPEAN DIRECIVE 2001/20/EC on the approximation of the laws, regulations and administrative provisions of the Member State relating to the implementation of good clinical practice in the conduct. Of clinical trials on medicinal products for human use, Off J Europ Communities, L121/34-44, 2001.

[18] Note for Guidance on Good Clinical Practice, ICH E6GCP96, EMEA, 2002: 1-55.

[19] World Medical Association declaration of Helsinky. Ethical Principles for Medical Research Involving Human subjects, amended until 2004

[20] Rosado C, Rodrigues LM. In vivo study of the physiological impact of stratum corneum sampling methods. Int J Cosmet Sci. 2003; 25(1-2):37-44

[21] Bashir SJ, Chew A, Anigbogu A, Dreher F, Maibach HI, Physical and physiological effects of stratm corneum tape stripping, S Res and Techn 2001; 7:40-48

[22] Ohman H, Vahlquist A. In viv studies concerning a $\mathrm{pH}$ gradient in human stratum corneum and upper pidermis. Acta Derm Venerol (Stockh) 1994;4:375-379. [23] Pereira MM, Saraiva J. Rodrigues LM. A utilização da biopsia superficial cutânea como modelo de estudo in vivo da lesão cutânea. Revista Lusófona de Ciência Tecnologias da saúde 2010;2:218-224.
[24] Seidenari S, Giusti F, Pellacani G. Instrumenta Evaluation of Occluded Patch Test Reactions. In: Serup J, Jemec G, Grove G, eds. Handbook Non-Invasive Methods and the Skin. 2nd ed. Texas: C.H.I.P.S; 2006 973-979.

[25] Rogiers V; EEMCO Group. EEMCO guidance for the assessment of transepidermal water loss in cosmetic sciences. Skin Pharmacol Appl Skin Physiol 2001;14 (2): $117-28$

[26]Takiwaki H, Serup J. Measurement of erytema and melanin indices. In: Serup J, Jemec G, Grove G, eds.

Handbook Non-Invasive Methods and the Skin. 2nd ed. Texas: C.H.I.P.S.; 2006: 377-383

[27] Berardesca E, Lévêque JL, Masson P. EEMCO Guidance for the Measurement of $\mathrm{Skin}$ Microcirculation. Skin Pharmacol Appl Skin Physio 2002;15 (6): 442-456

[28] Bircher A et al. Guidelines formeasurement of cutaneous blood flow by laser Doppler flowmwtry. Contact Dermatitis 1994; 30: 65-72.

[29] Bernardi L, Berardesca E. Measurement of Skin Blood Flow by Laser-Doppler Flowmetry. In:

Berardesca $\mathrm{E}$ et al, eds. Bioengineering of the skin: Methods and instrumentation. 2nd ed. Texas: C.H.I.PS; 2006: 13-27.

[30] Graham JS et al. Bioengineering methods employed in the study of wound healing of sulphur mustard burns. Skin Res Technol. 2002; 8(1):57-69

[31] Belcaro G, Nicolaides A. Laser Dopple Flowmetry: Principles of Technology and Clinica Applications. In: Serup J, Jemec G, Grove G, eds. Handbook Non-Invasive Methods and the Skin. 2nd ed. Texas: C.H.I.P.S; 2006: 709-715

[32]Choi C, Bennett R. Laser Dopplers to Determine Cutaneous Blood Flow. Dermatol Surg 2003;29 (3): $272-80$. 
Biomedical and

Biopharmaceutical

Research

Jornal de Investigacãa
Biomédica e Biofarmacêutica

) 